\title{
A Temporal-BRDF Model-Based Approach to Change Detection
}

\author{
L.Rebelo and P. Lewis \\ Department of Geography \\ University College London \\ WC1H 0AP, UK \\ Email: 1rebelo@geog.ucl.ac.uk
}

\author{
D.P.Roy \\ Department of Geography \\ University of Maryland and \\ Goddard Space Flight Centre \\ Code 922 (B32), Greenbelt, MD 20771 \\ USA
}

\begin{abstract}
Remote Sensing provides the only practical means to monitor changes over large areas. This paper describes the development of a generic algorithm designed to map the temporal occurrence and spatial extent of areas exhibiting sudden change. The algorithm is demonstrated here applied to the problem of mapping fire affected areas. The research further develops the work of [1], which implemented a bi-directional reflectance (BRDF) model-based change detection algorithm to map the approximate day and location of burning, using daily $500 \mathrm{~m}$ MODIS surface reflectance data. An original algorithm assumption is that the surface state remains static prior to the changes of interest. This is problematic in the presence of underlying change (for example, due to vegetation phenology) especially when there are missing and/or cloudy data. In an attempt to deal with this issue, an additional kernel has been added to the BRDF model in the form of a cubic function of time. In addition, a step function kernel has been introduced in order to more robustly detect step-like changes. These modifications and preliminary results over southern Africa using daily MODIS land surface reflectance data are presented.
\end{abstract}

\section{INTRODUCTION}

The remote sensing of changes taking place on the earth's surface forms an extremely active research field, with results forming an input to models of the global climate, biogeochemical and hydrological cycles. Vegetation fires have an impact on these cycles, and the identification and delineation of fire affected areas may be seen as a change detection problem. A number of change detection methods have been proposed based upon statistical classification approaches. With the availability of well calibrated and geo-located satellite data more physically based approaches are being developed. The research described develops the algorithm of [1] who implemented a new, generic approach to change detection. A model describing the anisotropic nature of surface reflectance was inverted over a time series of MODIS reflectance data for an area of southern Africa, and the resulting model parameters were used to provide a subsequent prediction of reflectance. Areas of surface change are identified as large discrepancies between the observed and predicted reflectances. Different types of changes may be separated using a temporal consistency threshold, as well as by examining the magnitude and the sign difference of potential 'change' pixels. Although results indicate that this algorithm is successful in mapping both the location and the approximate day of burning, problems occur in the presence of underlying phenological changes and various heuristics are required to separate changes of interest (i.e. burning) from these changes.

\section{Methodology}

It is widely accepted that the reflectance of solar radiation from the earth's surface is highly anisotropic. This variation in reflectance as a function of the geometry at which the surface is illuminated at and viewed from may be described by a Bi-Directional Reflectance Distribution (BRDF) model. The model used in the current research and that of [1] is the semiempirical kernel driven approach of [2]. The surface BRDF is modeled as a linear combination of kernels which are functions of illumination and viewing angles only. Three kernels have been shown to describe sufficiently the BRDF shapes of most naturally occurring surfaces [3]. These typically consist of i) an isotropic kernel to describe isotropic scattering from the surface, ii) a volumetric kernel which provides a single scattering approximation to radiative transfer, and iii) a geometic kernel to account for shadowing effects. The model thus takes the form of Equation 1.

$$
\rho\left(\lambda, \Omega, \Omega^{\prime}\right)=f_{\text {iso }}(\lambda)+f_{\text {vol }}(\lambda) k_{\text {vol }}\left(\Omega, \Omega^{\prime}\right)+f_{\text {geo }}(\lambda) k_{\text {geo }}\left(\Omega, \Omega^{\prime}\right)
$$

where $\rho$ is the spectral reflectance, $\Omega$ and $\Omega^{\prime}$ represent the viewing and illumination vectors respectively, and $k_{v o l}$ and $k_{g e o}$ are the kernels. These are geometric expressions of BRDF shapes and are functions of viewing and illumination geometry only. $f_{i s o}, f_{v o l}$, and $f_{g e o}$ are the kernel weights which describe the relative contribution of each type of scattering.

In the original implementation of the change detection algorithm [1] the model was inverted over a 16 day sliding window. An assumption of this approach is that the surface state remains invariant over the size of the window. With too large a window the results may be smoothed and the probability of underlying change occurring increased, while a small window size may result in larger uncertainties. Either of these may be problematic when looking for sudden changes.

In order to address this issue a polynomial temporal model has been introduced to the isotropic term of the BRDF model (Equation 1), as a cubic function of time. A major assumption of this approach is that the BRDF shape parameters remain 
constant over the time period of the inversion. The model now takes the form of Equation 2.

$$
\begin{aligned}
& \rho\left(\lambda, \Omega, \Omega^{\prime}\right)= \\
& f_{\text {iso }_{(0)}}(\lambda)+f_{\text {iso }_{(1)}}(\lambda) t+f_{\text {iso }_{(2)}}(\lambda) t^{2}+f_{\text {iso }_{(3)}}(\lambda) t^{3}+ \\
& f_{\text {vol }}(\lambda) k_{\text {vol }}\left(\Omega, \Omega^{\prime}\right)+f_{\text {geo }}(\lambda) k_{\text {geo }}\left(\Omega, \Omega^{\prime}\right)
\end{aligned}
$$

Previously the model parameters were updated for each new observation of reflectance (calculated from the previous 16 days of observations). For the temporal model however (Equation 2), only the three parameters $f_{\text {iso }}(\lambda) t, f_{\text {iso }}(\lambda) t^{2}$ and $f_{\text {iso }}(\lambda) t^{3}$ are allowed to vary as a function of time. Over the time period of the inversion the BRDF shape parameters $\left(f_{\text {vol }}(\lambda), f_{\text {geo }}(\lambda)\right)$ thus remain constant.

\section{Step Detection}

The occurrence of a fire typically results in a sudden decrease in MODIS band 2 (841-876 $\mathrm{nm})$, band 5 (1230$1250 \mathrm{~nm})$ and band $6(1628-1652 \mathrm{~nm})$ reflectances [1]. In order to model such changes, a 'step function kernel' has been incorporated into the model by the introduction of an additional parameter (Equation 3).

$$
a(\lambda, t)=s(\lambda) H_{c}(t)
$$

$a(\lambda, t)$ represents the change in brightness, $s(\lambda)$ the magnitude of the change, and $H_{c}(t)$ is a heaviside function, defined in Equation 4 , and $c$ is a non-linear parameter representing the day of the step change.

$$
H_{c}(t)= \begin{cases}0 & : t<c \\ 0.5 & : t=c \\ 1 & : t>c\end{cases}
$$

Parameter $c$ is inverted by stepping through each possible value of $c$ (each observation within the time series) and the remaining linear parameters are inverted using the method of least squares. For a generic change detection approach the 'best fit' value for $c$ may be found by selecting the value which gives the lowest error in model fit. The method used to select the most appropriate value of $c$ may be refined according to the changes of interest. In this case a measure has been defined to specifically locate 'burn type' changes (i.e. a step like drop in reflectance). This is discussed in more detail in section $\mathrm{V}$.

\section{Cloud Filtering}

Residual cloud and sub-pixel clouds are occasionally found in the MODIS 500m land surface reflectance data which have not been identified in the product quality assessment (QA) bits. If these are not removed from the temporal sequence then they will contribute to subsequent predictions of reflectance. A chi type measure is defined to investigate the probability of a new observation belonging to the same set as that used in the model inversion (Equation 5);

$$
Z=\frac{\rho_{\text {observed }}\left(\lambda, \Omega, \Omega^{\prime}\right)-\rho_{\text {predicted }}\left(\lambda, \Omega, \Omega^{\prime}\right)}{\varepsilon}
$$

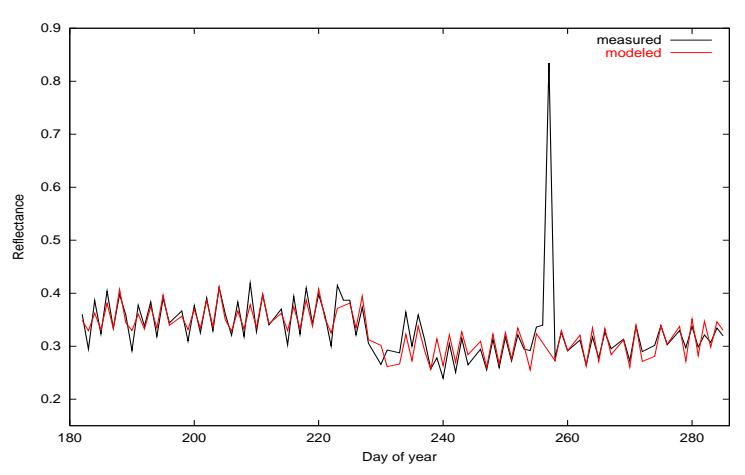

Fig. 1. Modeled and measured band 5 reflectance

where $\rho_{\text {observed }}\left(\lambda, \Omega, \Omega^{\prime}\right)$ is the observed reflectance, $\rho_{\text {predicted }}\left(\lambda, \Omega, \Omega^{\prime}\right)$ is the model predicted reflectance and $\varepsilon$ is the error in model prediction. This is calculated as;

$$
\varepsilon=e \sqrt{\left(1+\frac{1}{w}\right)}
$$

where $e$ is the expectation of error in an observation and $\frac{1}{w}$ is the 'weight of determination' [4]. The latter is defined as;

$$
\frac{1}{w}=[U]^{T}[M]^{-1}[U]
$$

where $[U]$ is the parameter vector and $[M]^{-1}$ is the inverse matrix, and ${ }^{T}$ denotes the transpose operation. $e$ is approximated from the residuals (Equation 8 ) where $m$ is the number of observations and $m-7$ the degrees of freedom.

$$
e^{2}=\frac{1}{m-7} \sum_{i=1}^{i=m}\left(\rho_{\text {observed }}\left(\lambda_{i} \Omega_{i} \Omega_{i}^{\prime}\right)-\rho_{\text {predicted }}\left(\lambda_{i} \Omega_{i} \Omega_{i}^{\prime}\right)\right)^{2}
$$

Multiple iterations are performed and isolated spikes (high positive values) are removed at each pass using the best knowledge of the parameter estimation and noise in the data at each iteration. Figure 1 shows the modeled and measured band 5 reflectance for a single pixel which burned on day of year 225. The inversion has been performed over a time series of 105 days. The sequence contains a spike on day 257 which is probably a cloud that has not been flagged by the product QA bits. The model clearly fits the observed reflectances well, with the minimum of the rmse (0.011) occurring on day 226. The isolated spike is identified by its high chi value (Equation 5 , and does not contribute to the subsequent predictions of reflectance.

\section{IDENTIFYING FIRE AFFECTED AREAS}

With the increase in cloud cover over parts of southern Africa towards the end of the year, and a decrease in the confidence of predicting a step at the ends of the temporal sequence, it is necessary to incorporate some measure relating to the confidence with which a step may be predicted at a certain point based on the sampling. The weight of determination (Equation 7) provides an indication of the uncertainty in prediction as a result of the angular and temporal sampling 


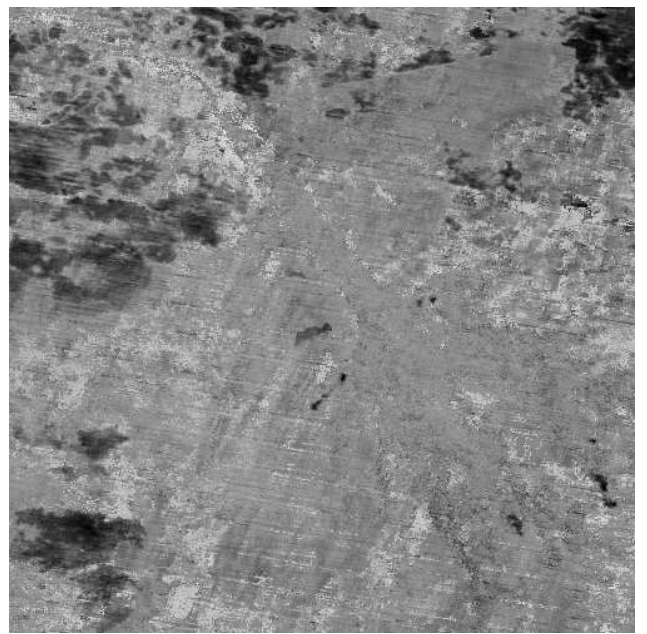

(a) Fire affected areas

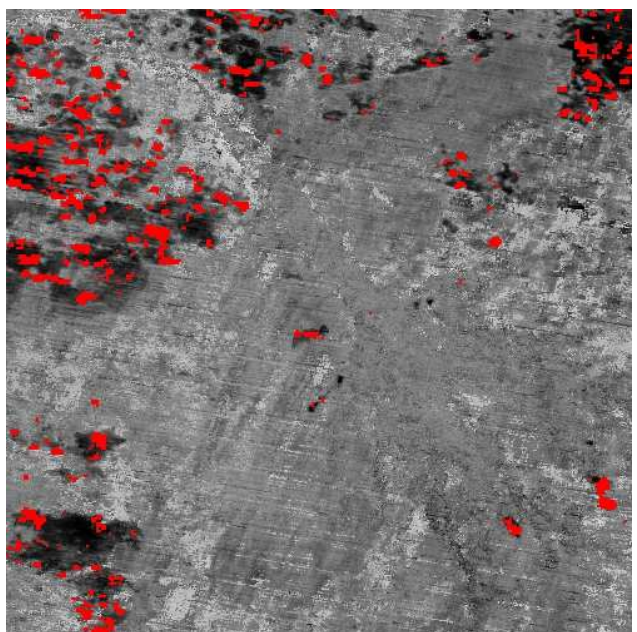

(b) Active fire pixels and burned areas

Fig. 4. Location of active fires and area affected by fire

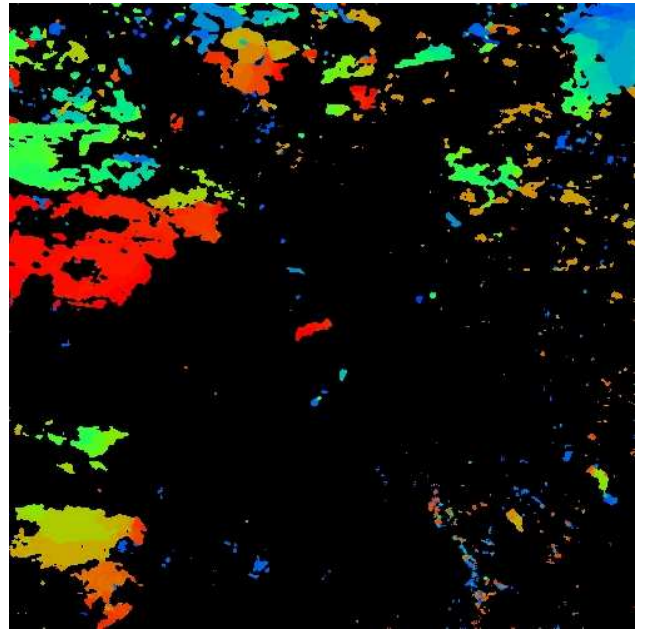

(a) Day of burn

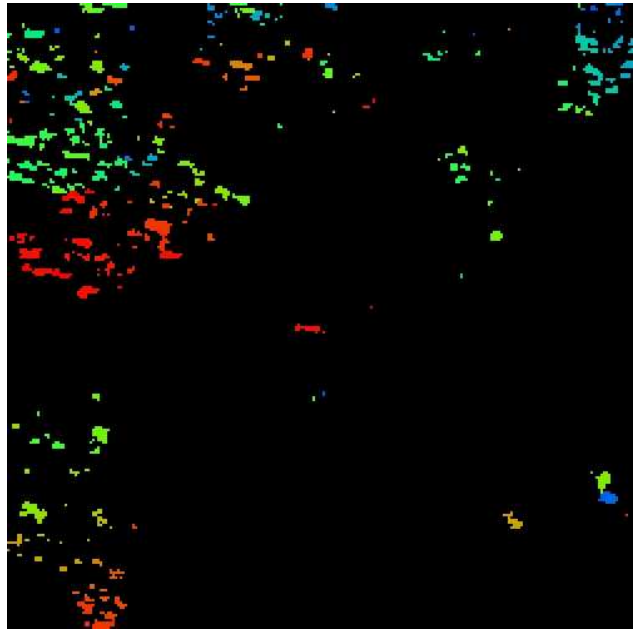

(b) Day of active fire

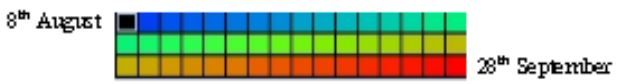

Fig. 5. Temporal location of fires

applicability of this method to different types of change, and a comparison with a more recent version of the MODIS burned area algorithm [5] will be investigated.

\section{REFERENCES}

[1] D. Roy, P. Lewis and C. Justice, (2002) Burned are mapping using multitemporal moderate spatial resolution data - a bi-directional reflectance model-based expectation approach, Remote Sensing of Environment(83), 263-286

[2] L.J. Roujean, M. Leroy and P.Y. Deschamps, (1992) A bi-directional reflectance model of the Earth's surface for the correction of remote sensing data, Journal of Geophysical Research(97), 20455-20468
[3] W. Lucht and J.L. Roujean, (2000) Considerations in the parametric modelling of BRDF and albedo from multiangular satellite sensor $o b-$ servations, Remote Sensing Reviews(18), 343-379

[4] W. Lucht and P. Lewis, (2000) Theoretical noise sensitivity of BRDF and albedo retrieval from the EOS-MODIS and MISR sensors with respect to angular sampling, International Journal of Remote Sensing(21), 81-98

[5] D. Roy, Y. Yin, P. Lewis and C.O. Justice Prototyping a global algorithm for systematic fire-affected area mapping using MODIS time series data, In preparation

[6] E.F. Vermote, N.Z.El Saleous and C.O. Justice (2002)Operational atmospheric correction of the MODIS data in the visible to middle infrared: first results, Remote Sensing of Environment(83), 97-111 